\title{
A new design of laser phase-shift range finder independent of environmental conditions and thermal drift
}

\author{
Shahram Mohammad Nejad \\ Kiazand Fasihi \\ Department of Electrical Engineering, Iran University of Science and Technology (IUST), Tehran, Iran
}

\begin{abstract}
This paper presents a technique to improve the performance of laser phase-shift range finders. The phase measurement is performed by using a new method to extract the phase-shift data from the peak of received and transmitted intermediate frequency signal amplitudes. The pulse width modulation is used to minimize the noise effect and enhance the signal amplitude measurement. The advantages of proposed system over others are its ability of proper isolation, which reduces crosstalk, and independency of frequency thermal drift. Theoretical calculations and experimental results have shown the accuracy better than $2 \mathrm{~mm}$ over measuring distance range of $0.3 m-163 m$.
\end{abstract}

Keywords: crosstalk, frequency thermal drift, laser range finding, phase shift, pulse width modulation.

\section{Introduction}

Laser range finders are suited for industrial applications and the systems based on semiconductor laser diodes are supposed to be rather common because their performances, small size, easy to drive and inexpensive. The measurement of the phase-shift between the transmitted and received signals that is combined with heterodyne technique is well adapted to medium range distance measurement, but is limited by the crosstalk occurring between the emitting and receiving circuits [1-5] and the frequency thermal drift. The presented method is a solution to improve the performance of laser range finders.

\section{Basic principles modified phase-shift measurement method}

The basic operation of laser phase-shift range finder is based on amplitude sine-wave modulation of laser intensity and conversion of the flight time due to propagation into phaseshift. If $f_{0}$ is the modulation frequency, the measured phase-shift $\Delta \phi$, between the optical signal reflected from the target and the reference signal emitted by the laser diode, permit the determination of distance $d$, according to following relationship [1]:

$\Delta \phi=\omega_{1} \tau_{1}=4 \pi f_{0} \frac{d}{c}$
It is important to have a good resolution for the phase measurement which may be achieved by heterodyne phase detection technique. By using the heterodyne technique, the phase-shift can be written as $\Delta \varphi=2 \pi T_{X} / T_{I F}$, where $T_{I F}$ is the period of intermediate frequency signal and $T_{X}$ is the time corresponding to high pulse width of rectangular wave train that is obtained at the output of phase-shift detector circuit. So the distance to the target $d$ can be determined by the expression [2]:

$d=\frac{\lambda_{0}}{2} \frac{T_{X}}{T_{I F}}$

The accuracy in the phase-shift measurement is affected by several factors such as the crosstalk effect, the accuracy and stability of signal frequency of laser diode driver, the local frequency and the clock pulse frequency of the phase detector counter. We present a different method for phaseshift measurement so that the sensitivity to called parameters will be minimized. In order to describe our method, we express the signal of the laser diode driver as:

$S_{D}(t)=K_{1} \cos \left(\omega_{0} t+\varphi_{1}\right)$

where $K_{1}$ and $\omega_{0}$ are the amplitude and angular frequency of the laser diode driver signal, respectively, and $\varphi_{1}$ is its initial phase. The received signal after passing through an AGC circuit can be expressed as:

$S_{R}(t)=K_{2} \cos \left(\omega_{0} t+\varphi_{2}\right)$

where $K_{2}$ and $\omega_{0}$ are the amplitude and angular frequency of the received signal and $\varphi_{2}$ is its initial phase. We define the local frequency by the following expression:

$S_{L}(t)=K_{3} \cos \left(\left(\omega_{0} \pm \omega_{I F}\right) t+\varphi_{0}\right)$

where $\omega_{I F}$ is the intermediate angular frequency and $K_{3}, \omega_{0}$ and $\varphi_{0}$ indicate similar parameter defined above. Multiplying the local frequency by the signal of laser diode driver and the received signal, and rearranging the results, leads to following equation: 


$$
\begin{aligned}
S_{L D}= & \frac{K_{1} K_{3}}{2}\left\{\cos \left[\left(2 \omega_{0} \pm \omega_{I F}\right) t+\varphi_{1}+\varphi_{0}\right]\right. \\
& \left.+\cos \left(\mp \omega_{I F} t+\varphi_{1}-\varphi_{0}\right)\right\}
\end{aligned}
$$

and

$$
\begin{aligned}
S_{L R} & =\frac{K_{1} K_{3}}{2}\left\{\cos \left[\left(2 \omega_{0} \pm \omega_{I F}\right) t+\varphi_{2}+\varphi_{0}\right]\right. \\
& \left.+\cos \left(\mp \omega_{I F} t+\varphi_{2}-\varphi_{0}\right)\right\}
\end{aligned}
$$

A multiplier IC can be used to multiply these signals. With the low pass filtering of these signals, the output of low pass filter can be expressed as:

$$
\begin{aligned}
& \frac{K}{2} \cos \left(-\omega_{I F} t+\varphi_{1}-\varphi_{0}\right)+\frac{K}{2} \cos \left(\omega_{I F} t+\varphi_{1}-\varphi_{0}\right) \\
& =K \cos \left(\varphi_{1}-\varphi_{0}\right) \times \cos \left(\omega_{I F} t\right)
\end{aligned}
$$

And

$$
\begin{aligned}
& \frac{K^{\prime}}{2} \cos \left(-\omega_{I F} t+\varphi_{2}-\varphi_{0}\right)+\frac{K^{\prime}}{2} \cos \left(\omega_{I F} t+\varphi_{2}-\varphi_{0}\right) \\
& =K^{\prime} \cos \left(\varphi_{2}-\varphi_{0}\right) \times \cos \left(\omega_{I F} t\right)
\end{aligned}
$$

Where $K$ and $K^{\prime}$ correspond to $K_{1} K_{3}$ and $K_{2} K_{3}$, respectively. We use a peak detector circuit to extract the peak amplitudes of signals that contain the phase data. In section 3.2, we introduce a new technique that make signal amplitude measurement independent of $K$ and $K^{\prime}$. The amounts of $\left(\varphi_{1}-\varphi_{0}\right)$ and $\left(\varphi_{2}-\varphi_{0}\right)$ are measured in processing unit. Finally, the phase-shift that corresponds to the propagation of laser beam, can be calculated.

\section{Design of modified laser phase-shift range finder}

Fig.1 shows the main parts of the modified laser range finder. The system is composed of four main blocks that each of them will be described as follow.

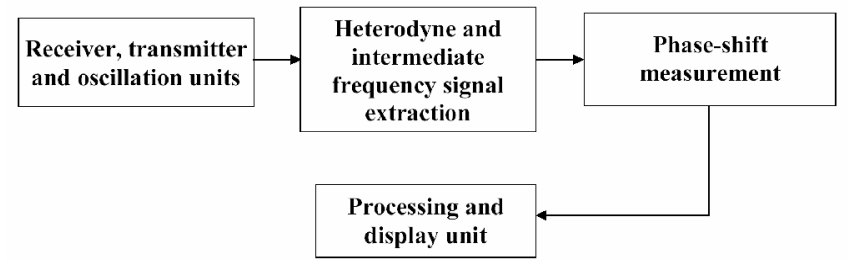

Fig. 1: The block diagram of the modified laser range finder.

\subsection{The unites of emission, receiver and oscillation}

The first block is composed of three main electrically isolated units. The laser diode amplitude is modulated by a sine-wave signal with frequency of $230 \mathrm{kHz}$, so the unambiguous range with respect to frequency of modulation is about $163 \mathrm{~m}$. The amplitude modulation index is given about $2 \%$ to decrease the crosstalk effect. The main device in oscillation unit is a $66.240 \mathrm{MHz}$ crystal oscillator that is thermally compensated. We use the divider circuits to produce $230 \mathrm{kHz}$ and $207 \mathrm{~Hz}$ frequencies that are the frequencies of the laser diode driver and the intermediate signal, respectively. A multiplier IC is used to mix the main harmonics of mentioned frequencies and produce the local frequencies. The block diagram of emission and oscillation units is shown in Fig.2.

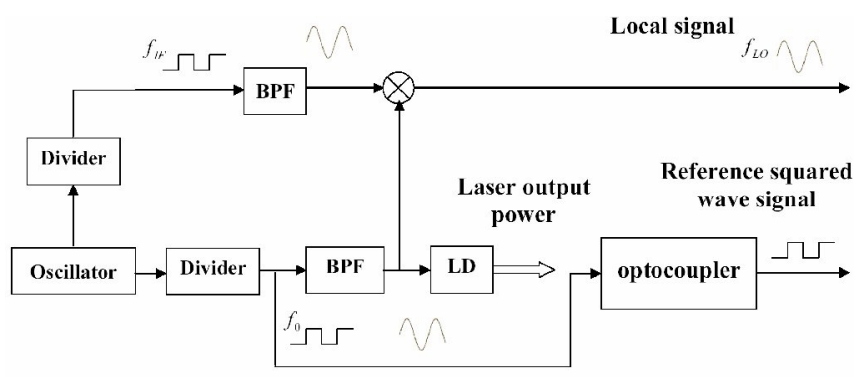

Fig. 2: The block diagram of emission and oscillation units.

The block diagram of the receiver unit is illustrated in Fig.3. The receiver's Front-End is a transimpedance amplifier with $1.5 \mathrm{MHz}$ bandwidth [6,7]. The Front-End converts the incident modulated laser beam to an electrical signal. The detected signal passes through a preamp circuit and after band pass filtering enters to an AGC circuit. The crosstalk between the transmitter and the receiver circuits is the main limitation factor that doesn't permit an accurate predication of the phase-shift measurement. Hence in order to minimize the crosstalk effect, it is necessary to isolate receiver and transmitter circuits. We convert the reference and received signals to square wave signals with passing them through a limiter circuit. These signals then pass through TTL compatible optocouplers before transferring to other units. The experimental results show considerable decrease in crosstalk effect. The block diagram of the receiver circuit is shown in Fig.3.

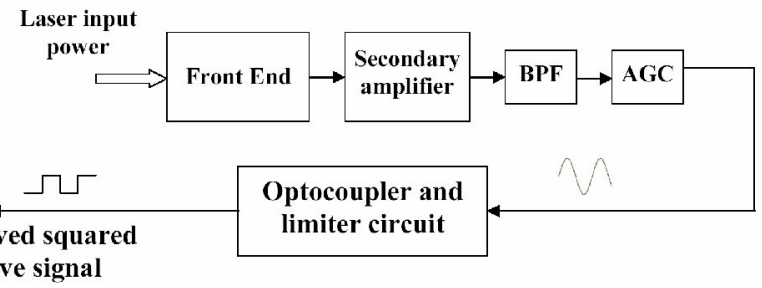

Fig. 3: The block diagram of the receiver unit.

\subsection{The heterodyne method and independency from environmental conditions}

In order to have a good resolution for the phase-shift measurement, it is necessary to use the heterodyne phase detection technique. In this technique each of transmitted and received signals, which are converted to square wave signals, are mixed with a local signal. The frequency of local signal is $\left(\omega \pm \omega_{I F}\right)$ and is obtained by mixing of reference and intermediate signals in the oscillation unit (see Fig.2). 
After low pass filtering the outputs of mixers (as illustrate in Fig.4), we will obtain signals with intermediate frequency based on (8) and (9). The amplitudes of these intermediate signals contain the phase data that should be extracted and processed. The equations (8) and (9) indicate that the amplitude of measured signal should be independent of $K$ and $K^{\prime}$. Fig.4 shows the proposed configuration to eliminate the measured phase dependency on the above parameters.

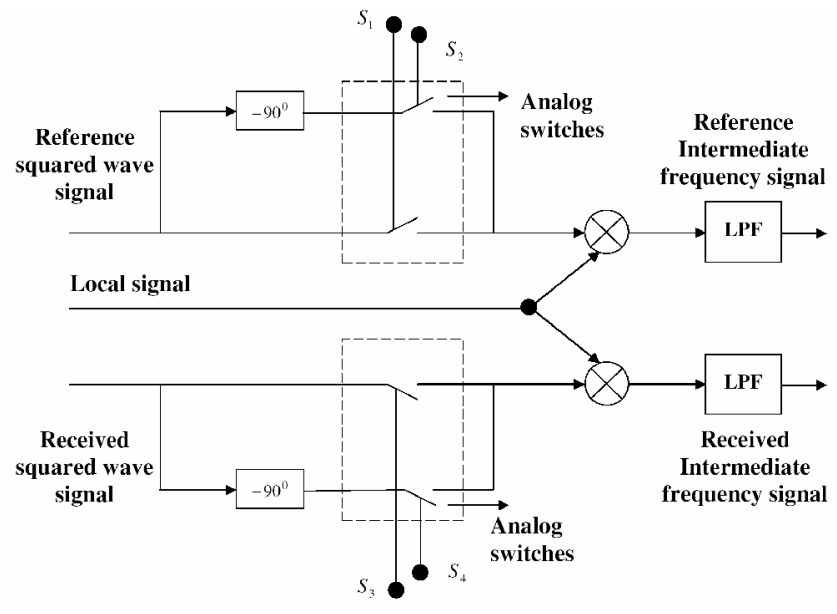

Fig. 4: The modified heterodyne technique.

In the proposed configuration, when the analog switch $S_{2}$ is activated, the local signal will be mixed with reference signal. In this case, after low pass filtering the resulting signal at $f_{I F}$ frequency, the intermediate signal can be written as:

$S_{I F_{1}}=K G_{L P F} \cos \left(\varphi_{1}-\varphi_{0}\right) \times \cos \left(\omega_{I F} t\right)$

where $G_{L P F}$ is the gain of active low pass filter. In the same way, if switch $S_{1}$ is activated, we will obtain the following signal:

$S_{I F_{2}}=K G_{L P F} \cos \left(\varphi_{1}-\varphi_{0}-90\right) \times \cos \left(\omega_{I F} t\right)$

In recent case, the reference signal passes initially through a unity gain $-90^{\circ}$ phase-shifter, before mixing with local signal. In either of the above cases, the amplitude of intermediate frequency is measured. By dividing the measured signal amplitudes to each other, we will have $\operatorname{tg}\left(\varphi_{1}-\varphi_{0}\right)$ that is independent from $K$ and $G_{L P F}$ parameters. In similar way, by activating $S_{3}$ and $S_{4}$ analog switches, we will have $\operatorname{tg}\left(\varphi_{2}-\varphi_{0}\right)$. Finally the amount of phase-shift $\left(\varphi_{2}-\varphi_{1}\right)$ can be calculated in processing unit. This method is a way to independency from environmental conditions in phase-shift measurement. We use an active all pass filter to implement a unity gain $-90^{\circ}$ phase-shifter. It must be noticed that in this method, the unique phase-shift measurement is limited to $90^{\circ}$, so the no ambiguity range is restricted to $\lambda_{0} / 8$.

\subsection{The Pulse Width Modulation for phase-shift data extraction}

In this section, we describe designed circuit for phaseshift extraction. As referred in the previous section, in the design, the peak amplitude of intermediate signal would contain the phase-shift data, so a peak detector is used to measure its value. We use a fast RMS to DC converter to implement this circuit. The output of this circuit is linearly proportional to the peak of sinusoidal intermediate frequency signal. In order to achieve an accurate DC signal measurement and minimize the noise effects, we use the PWM modulation. The block diagram of Fig. 5 shows the structure of the designed circuit for accurate DC signal measurement.

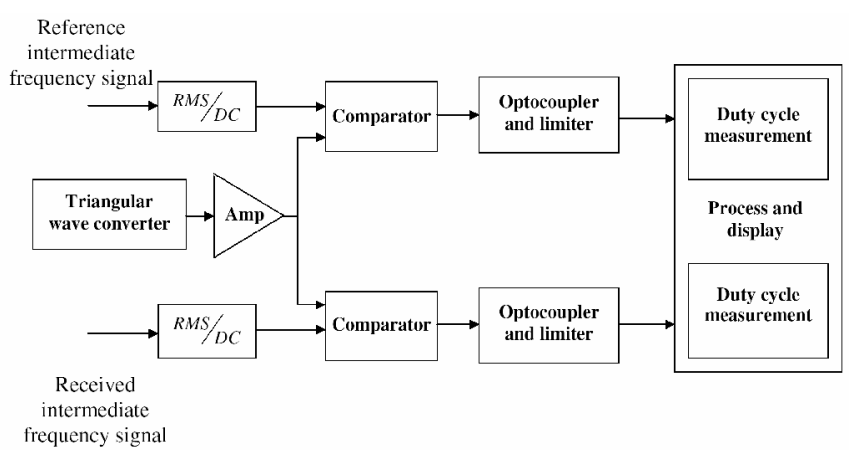

Fig. 5: The block diagram of the designed circuit for accurate DC signal measurement.

As shown in Fig.5, the amplitude of a triangular wave signal is adjusted to a desired level and compared with a DC signal in a fast comparator circuit. The amplitude of the triangular wave signal must be greater than the maximum amplitude of DC signals. The outputs of the comparator are square wave signals which have duty cycles corresponding to the DC signal amplitudes. The typical frequency of the triangular wave signal is about $200 \mathrm{~Hz}$, that provides the ability of averaging to increase the accuracy in the duty cycle measurement. The experimental results show an accuracy of $\pm 100 \mu \mathrm{V}$ in DC signals measurement. Since the maximum amplitude of intermediate frequency signal is adjusted at $8 \mathrm{~V}$, the $\pm 100 \mu \mathrm{V}$ accuracy in DC signal measurement corresponds to a resolution of $\pm 2 \mathrm{~mm}$ over the measuring distance range of $0.3 m-163 m$.

\subsection{The processing unit}

In this unit, the duty cycles of reference and received signals that contain phase-shift data are measured by the processor. We can express the phase-shift in accordance with measured duty cycles as:

$$
V=V_{m}(2 \cdot D u-1)
$$

Where $V_{m}$ and $D u$ are the peak amplitude values of triangular wave form and measured duty cycle, respectively. It can be shown that the measured phase-shift is frequencyindependent. This leads to remove the phase error due to the 
frequency thermal drift. The value of phase error due to the electronic delay time is constant and can be eliminated by system calibration. The range finder measures the target range relative to a reference range. The accuracy of the measurement depends on the accuracy of the reference range. If $\Delta \varphi_{R E F}$ is the phase-shift corresponding to the reference range $R_{R E F}$, then the calibrated range is given by the equation [5]:

$R=R_{R E F}+\frac{c}{4 \pi f_{0}}\left(\Delta \varphi-\Delta \varphi_{R E F}\right)$

where $c$ is the speed of light in vacuum, $f_{0}$ is the frequency of laser modulation signal, and $\Delta \varphi$ is the measured phaseshift. In the processing unit, the amounts of phase-shift and the target range calculated with respect to (12) and (13).

\section{The thermal drift error comparison}

In the common laser phase-shift range finders, the accuracy of phase-shift measurement depends on the accuracy and the stability of intermediate frequency, the frequency of laser driver signal and the clock pulse of the counter. If we define $T_{X}=N T_{\text {count }}$ in (2), where $T_{\text {count }}$ and $N$ are the period of counters clock pulse and the number of counted pulses, respectively, then (2) can be rewritten as:

$d=\frac{T_{x}}{T}\left(\frac{\lambda_{0}}{2}\right)=\frac{f_{I F}}{f_{\text {clock }}} \frac{\lambda_{0}}{2} N$

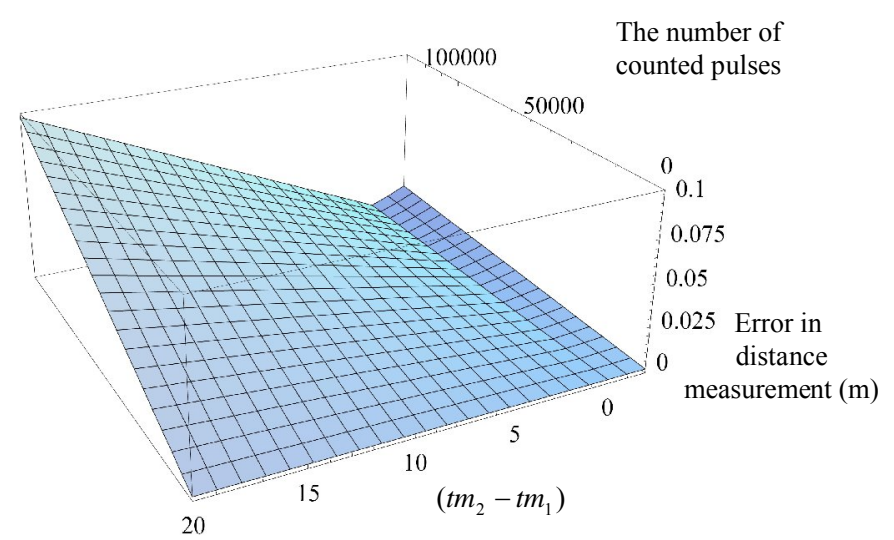

Fig. 6: The diagram of simulated distance measurement error due to the difference in thermal drift of crystal frequencies in a distance range of $0 m-163 m$.

If we neglect the error due to the thermal drift in $f_{\text {clock }}$, the total frequency thermal drift can be written as:

$$
\Delta x=\frac{\Delta f_{I F}}{f_{\text {count }}} \frac{\lambda N}{2}-\frac{f_{I F}}{f_{\text {count }}} \frac{c \Delta f_{1}}{2 f_{1}^{2}} N
$$

Where $\Delta f_{I F}=f_{I F}\left(\frac{t m_{1}}{60}\right)+f_{1}\left(\frac{t m_{2}-t m_{1}}{60}\right)$ and $\Delta f_{1}=f_{1}\left(\frac{t m_{1}}{60}\right)$ are frequency thermal drifts versus temperature in laser driver signal and intermediate signal, respectively, and $\mathrm{tm}$ is the temperature stability in the crystal frequency over $60^{\circ}$ temperature variation range.

The distance measurement error in a distance range of $0 m-163 m$, is simulated and shown in Fig.6. In this simulation it has been assumed that $t m_{1}=2 \mathrm{ppm}$ and $\left(t m_{2}-t m_{1}\right)$ varies from $-2 \mathrm{ppm}$ to $20 \mathrm{ppm}$. In addition, the frequency of laser driver signal, intermediate frequency and the counters clock pulse frequency are taken as $f_{1}=247 \mathrm{KHz}, f_{I F}=200 \mathrm{~Hz}, \quad$ and $f_{\text {count }}=66.240 \mathrm{MHz}$, respectively. It must be noted that the maximum number of the clock pulses that is counted with respect to the period of intermediate frequency signal and the counters frequency, is equal to 123000 . The worst-case analysis shows that the measured error due to thermal drift in crystals frequencies, assuming of $t m_{1}=2 \mathrm{ppm}$ and $\mathrm{tm}_{2}=4 \mathrm{ppm}$, is equal to $19.6 \mathrm{~mm}$, which is considerable. The new designed system is independent of the thermal drift of frequency sources, because the amplitudes of the intermediate signal duty cycles contain phase-shift data.

\section{REFERENCES}

[1] T.Bosch and M. Lescure, "Crosstalk Analysis of $1 m$ to $10 m$ Laser Phase-shift Range Finder", IEEE Transaction on Instrumentation and Measurement, Vol. 46, No. 6, Dec. 1997.

[2] S. Poujouly and B. Journet, "Laser Range-Finder by Phase-shift Measurement: Moving Toward Smart Systems", Proc. SPIE, Vol. 4189, pp. 152-160, 2001.

[3] B. Journet, G. Bazin and C. Durieu, "An Unique Certain to Estimate the Performances of Some Laser Range Finders", SPIE, Vol. 4420, pp.32-41, 2001.

[4] B. Journet, G .Bazin, F. Bars, "Conception of an Adaptive Laser Range Finder Based on Phase-shift Measurement”, IEEE, pp. 789789, 1996.

[5] K. S .Hashemian, P. T. Hurst and J. N. Oliver, "Source of Error in Laser Range Finder, Rev. Sci. Instrum”, Vol. 65, No. 10, pp. 31653171, Oct. 1994

[6] S. B. Alexander, "Optical Communication Receiver Design”, First Edition, SPIE, 1997.

[7] J. G. Graeme, "Photodiode Amplifier Op-Amp Solution", McGraw Hill. 